\title{
Conjugation of Ginsenoside Rg3 with Gold Nanoparticles
}

\author{
Youmie Park, ${ }^{\star}$ A-Rang Im, ${ }^{\dagger}$ Eun Ji Joo, ${ }^{\dagger}$ Jihye Lee, ${ }^{\ddagger}$ Hyeung-geun Park,${ }^{\ddagger}$ Young-Hwa Kang,, \\ Robert J. Linhardt, ${ }^{\#}$ and Yeong Shik Kim
}

\author{
College of Pharmacy, Inje University, 607 Obang-dong, Gimhae, Gyeongnam 621-749, Korea. *E-mail: youmiep@inje.ac.kr \\ ${ }^{\dagger}$ Natural Products Research Institute, College of Pharmacy, Seoul National University, Seoul 151-742, Korea \\ ${ }^{\star}$ Research Institute of Pharmaceutical Sciences and College of Pharmacy, Seoul National University, Seoul 151-742, Korea \\ ${ }^{\S}$ Division of Applied Biosciences, College of Agriculture \& Life Sciences, Kyungpook National University, \\ Daegu 702-701, Korea \\ ${ }^{*}$ Departments of Chemistry and Chemical Biology and Chemical and Biological Engineering, Center for Biotechnology \\ and Interdisciplinary Studies, Rensselaer Polytechnic Institute, 110 8th Street, Troy, NY 12180, USA \\ Received July 5, 2010, Accepted October 17, 2010
}

\begin{abstract}
Ginsenoside Rg3 was reported to have important biological activities. We demonstrate conjugation and quantification procedures of ginsenoside $\operatorname{Rg} 3$ to gold nanoparticles for future biological and medical applications. Ginsenoside $\operatorname{Rg} 3$ was conjugated to spherical gold nanoparticles using a bifunctional heptaethylene glycol linker. The sulfhydryl group of heptaethylene glycol was adsorbed onto gold nanoparticles, and carboxylic acid end of heptaethylene glycol was bonded through a hydroxyl group of $\operatorname{Rg} 3$ via ester bond formation. The conjugation of $\operatorname{Rg} 3$ was characterized with various spectroscopic techniques, high resolution-transmission electron microscopy, and using Rg3 monoclonal antibody. The Rg3- functionalized gold nanoparticles were $4.7 \pm 1.0 \mathrm{~nm}$ in diameter with a surface charge of $-4.12 \mathrm{mV}$. The total number of $\mathrm{Rg} 3$ molecules conjugated to a $3.6 \mathrm{~mL}$ solution of gold nanoparticle was determined to be $9.5 \times$ $10^{14}$ corresponding to $\sim 6$ molecules of $\mathrm{Rg} 3 /$ gold nanoparticle. These results suggest that ginsenoside $\operatorname{Rg} 3$ is successfully conjugated to gold nanoparticles via heptaethylene glycol linker. The quantification was performed by using $\operatorname{Rg} 3$ monoclonal antibody without interference of gold's intrinsic color.
\end{abstract}

Key Words: Ginsenoside Rg3, Gold nanoparticles, Rg3 monoclonal antibody, Conjugation, Nano-sized delivery vehicle

\section{Introduction}

Nano-sized metal particles have recently elicited great interest in many fields due to their novel potential applications. In particular, gold nanoparticles (AuNPs), also known as gold colloids, have held promise as nano-sized vehicles in the delivery of anti-cancer drugs. The most common means of preparing gold nanoparticles is the chemical reduction of $\mathrm{HAuCl}_{4}$ by sodium citrate or sodium borohydride. ${ }^{1}$ Gold colloids are very popular as delivery vehicles due to their straightforward synthesis, low cytotoxicity, biological compatibility, high tissue permeability, spherical shape with uniform size, and facile surface modifications. ${ }^{2}$ The surface of AuNPs has a characteristic binding affinity to thiols, amines, and disulfides, which facilitates their surface modification with ligands through either covalent linkage or strong physical adsorption. ${ }^{3}$ AuNPs have also been used in selective laser photothermal cancer therapy called hyperthermia. ${ }^{2,3}$ AuNPs have a special feature of very rapidly converting strongly absorbed light into thermal energy, which can be employed to selectively kill cancer cells. The labeling of AuNPs with biological ligands to specifically bind to desired cancer cells increases the effectiveness of thermal energy transfer to cancer cells without harming non-cancerous cells. ${ }^{3}$ However, photothermal cancer therapy is most effective in tissues close to skin and is only effective in small portions of these tissues. ${ }^{2}$ The introduction of polyethylene glycol (PEGylation) onto AuNPs affords a steric layer that reduces aggregation and minimizes opsonization and reticuloendothelial system clearance. PEGylation increases circulation half-life and provides colloidal stability under physiological salt concentrations. In addition, PEGylation increases the hydrophilicity of nanoparticles providing excellent water solubility and superior biocompatibility. For example, Gibson et al. used hexaethylene glycol to link between AuNPs and paclitaxel, resulting water soluble products. ${ }^{4}$ Bergen et al. reported that the modification of AuNPs surface with PEG-thiol or galactose-PEG-thiol afforded steric stabilization at physiological salt concentrations under conditions where unmodified AuNPs aggregated. ${ }^{5}$ In addition, PEG modification does not significantly affect the surface charge and size of AuNPs. Galactose-PEG-thiol ligand on AuNPs effectively resulted in hepatocyte targeted delivery in vivo. ${ }^{5}$ The influence of anchoring ligands and particle size on the colloidal stability of PEG-coated AuNPs has also been reported. ${ }^{6}$ This study suggests that AuNPs $(20 \mathrm{~nm})$ coated with thioctic acidterminated PEG (MW 5,000) are promising for potential drug delivery vehicles. Recently, Gu et al. reported nuclear penetration of PEG functionalized AuNPs, making these potentially useful in nuclear targeted drug delivery carrier. ${ }^{7}$

Anticancer drugs have been conjugated onto AuNPs to enhance their activities. Gibson et al. described $2 \mathrm{~nm}$ AuNPs covalently functionalized with an anti-cancer drug, paclitaxel. Their synthetic pathways involved the attachment of a flexible hexaethylene glycol linker at paclitaxel followed by coupling to phenol-terminated AuNPs. ${ }^{4}$ AuNPs functionalized with doxorubicin (adriamycin) were more effective in penetrating into the cell than doxorubicin alone. ${ }^{8}$ Methotrexate, inhibitor of dihydro- 
20(S)-Ginsenoside-Rg3

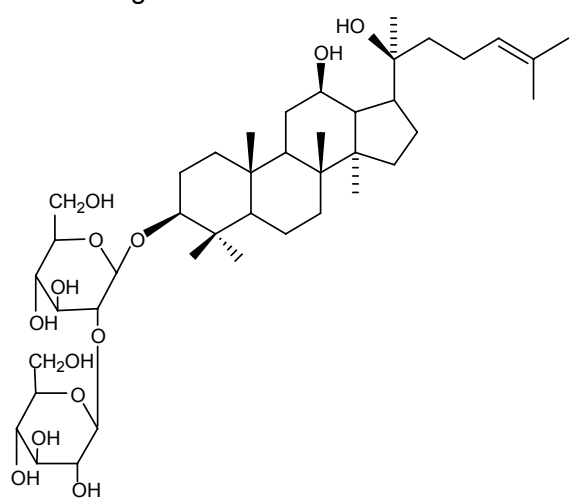

O-(2-carboxyethyl)-O'-(2-mercaptoethyl)heptaethylene glycol (HEG) $\mathrm{HS}-\mathrm{CH}_{2} \mathrm{CH}_{2}-\mathrm{OCH}_{2} \mathrm{CH}_{2} \frac{1}{7} \mathrm{OCH}_{2} \mathrm{CH}_{2}-\mathrm{COOH}$

Figure 1. Structures of Rg3 (20-(S)-ginsenoside-Rg3) and HEG used in this study.

folate reductase has also been conjugated to AuNPs. ${ }^{9}$ This study suggests that methotrexate-AuNPs may be more effective than free methotrexate for cancer treatment. Conjugating Kahalalide F (a marine cyclodepsipeptide isolated from a mollusk) with AuNPs improved the selective delivery into the cell and the activity of the compound. ${ }^{10}$

Natural products and their derivatives represent the majority of all anti-cancer drug candidates. Ginsenosides are a group of triterpenoid saponins found in Panax species (Panax ginseng C.A. Meyer, Araliaceae). So far more than 150 naturally occurring ginsenosides have been isolated from various parts of ginseng and these show diverse biological activities. Biological activities of ginsenosides include radical scavenging, vasodialating, neuroprotective, anti-viral, anti-obesity, and anti-cancer activities. Ginsenosides Rb1, Rb2, Rc, Rd, Rg1, and Re are major constituents of white and red ginseng, while ginsenosides $\mathrm{Rg} 3$, $\mathrm{Rg} 5, \mathrm{Rk} 1$, and F4 are known to be unique constituents of red ginseng. Many studies have been reported anticancer activities of ginsenoside $\mathrm{Rg} 3{ }^{11-16}$ Interestingly, ginsenoside-based nanoparticles composed of ginseng saponins, cholesterol, and phosphatidyl cholin upregulate both cellular and humoral immune response in mice. ${ }^{17}$

In this paper, we report conjugation of ginsenoside $\operatorname{Rg} 3$ to AuNPs using a heptaethylene glycol (HEG) linker. The final product, AuNPs-HEG-Rg3, was characterized using various spectroscopic techniques, high resolution-transmission electron microscopy (HR-TEM), zeta potential measurements, and enzyme-linked immunosorbent assay (ELISA) using Rg3 monoclonal antibody. The structures of Rg3 and HEG were shown in Figure 1.

\section{Experimental Section}

Synthesis of AuNPs-HEG. For the preparation of AuNPs$\mathrm{HEG}, \mathrm{HAuCl}_{4} \cdot 3 \mathrm{H}_{2} \mathrm{O}(4.5 \mathrm{mg})$ was dissolved in $80 \mathrm{~mL}$ water. Into this solution, $5 \mathrm{~mL}$ of sodium borohydride $(1 \mathrm{mg} / \mathrm{mL}$ concentration in water) was added drop by drop with stirring. As soon as the color of solution turned from pale yellow to orange- red, $O$-(2-carboxyethyl)-O'-(2-mercaptoethyl)heptaethylene glycol (HEG, structure in Figure 1) solution $(125 \mathrm{mg} / 1 \mathrm{~mL}$ water) was added drop by drop with stirring. For the preparation of bare AuNPs, the addition of HEG solution was omitted in the above procedure. UV-vis scanning (200 - $700 \mathrm{~nm}$ range) was recorded and high resolution-transmission electron microscopy (HR-TEM) was performed to observe morphology, size, and dispersion state of AuNPs-HEG. For a preparation of AuNPsHEG-Rg3, AuNPs-HEG solution ( $86 \mathrm{~mL}$ ) was concentrated by using centrifugal devices $(30 \mathrm{~K})$, and the volume was reduced, and further dried by nitrogen purging to make a final volume of $120 \mu \mathrm{L}$.

Conjugation of Rg3 with AuNPs-HEG. The concentrated AuNPs-HEG $(120 \mu \mathrm{L})$ was re-dissolved in $480 \mu \mathrm{L}$ of DMF. Into this solution, DIC $(8.9 \mu \mathrm{L})$ and DPTS $(5.7 \mathrm{mg})$ was added with stirring for $1 \mathrm{~h}$ at room temperature. Then, $2 \mathrm{mg}$ of $\mathrm{Rg} 3$ was added and the reaction was performed at room temperature overnight with stirring. After overnight incubation, water was added to make a DMF final concentration of $20 \%$. Then, excess reagents and $\mathrm{Rg} 3$ were removed by centrifugation using Nanosep omega $30 \mathrm{~K}(8,000 \mathrm{~g}$ force). The reaction mixture was washed exhaustively with $70 \%$ methanol $(12 \mathrm{~mL})$. As an alternative work-up, DMF concentration in the reaction mixture was adjusted to $10 \%$ with water and dialysis (MWCO 12,000 - 14,000 Da) was conducted as follows. The reaction mixture was sequentially dialyzed against water (one day, $2 \mathrm{~L}$ ), 50\% methanol (one day, $3 \mathrm{~L}$ ), and $70 \%$ methanol (two days, $2 \mathrm{~L} / \mathrm{a}$ day). The final volume was reduced to $3.6 \mathrm{~mL}$ by Nanosep omega $30 \mathrm{~K}(8,000 \mathrm{~g}$ force) and filtered through $0.2 \mu \mathrm{m}$ syringe filter (Pall Corporation, Acrosdisc ${ }^{\circledR}$ syringe filter) for analysis.

ELISA using Rg3 monoclonal antibody. The quantification of Rg3 on AuNPs-HEG-Rg3 was determined by direct ELISA method. AuNPs-HEG-Rg3 $(50 \mu \mathrm{L}$ out of the final volume 3.6 $\mathrm{mL}$ ) was serially diluted in $0.2 \mathrm{M}$ sodium carbonate (Sigma, St. Louis, MO, USA) buffer (coating buffer), and was adsorbed to the 96 well plate overnight at $4{ }^{\circ} \mathrm{C}$. As a negative control, AuNPs-HEG was also coated onto the plate by the same method above. The plate was blocked with 5\% BSA in tris-buffered saline containing $0.05 \%$ of Tween 20 (TBST) to reduce nonspecific adsorption and washed 3-times with TBST. The plate was reacted with the monoclonal antibody diluted in TBST at $37^{\circ} \mathrm{C}$ for $2 \mathrm{~h}$. After washing the plate with TBST 3-times, the monoclonal antibody to $\mathrm{Rg} 3$ was then reacted with goat antimouse IgG conjugated with horseradish peroxidase at $37{ }^{\circ} \mathrm{C}$ for $1 \mathrm{~h}$. After washing the plate with TBST 5-times, the substrate of ortho-phenylenediamine dihydrochloride was added to each well and incubated for $30 \mathrm{~min}$ at room temperature. After the incubation period, the absorbance of each well was read at 450 nm on UV-vis absorbance reader.

\section{Results and Discussion}

Surface plasmon resonance (SPR) showed a maximam of $515 \mathrm{~nm}$ and $521 \mathrm{~nm}$ for AuNPs and AuNPs-HEG, respectively (Figure 2). The adsorption of HEG to AuNPs provided water solubility as well as colloidal stability. Bare AuNPs easily aggregate in sodium phosphate buffer at $\mathrm{pH} 7.4$ during a stability test for 30 days, while AuNPs-HEG stays stable without notice- 


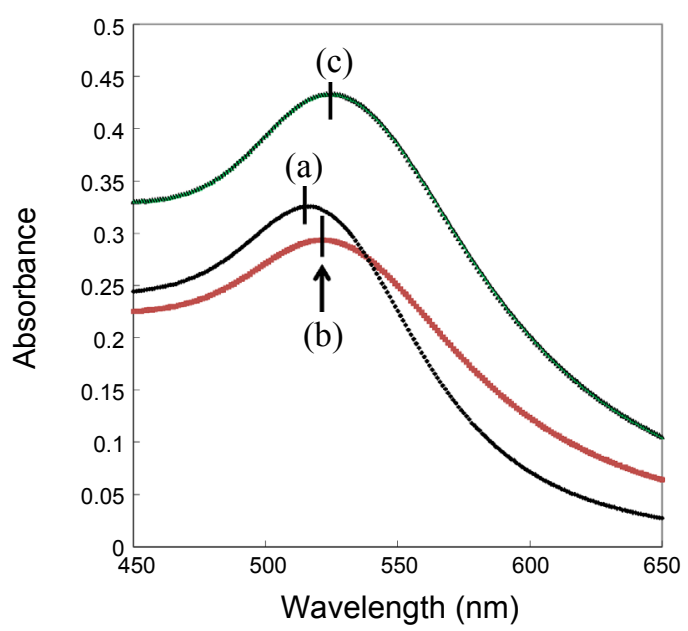

Figure 2. Surface plasmon resonance band of (a) AuNPs (515 nm), (b) AuNPs-HEG (521 nm), and (c) AuNPs-HEG-Rg3 (523 nm).

able aggregation (Figure S1). As shown in Figure S1, the color of bare AuNPs changed from red to violet-grey right after the addition of phosphate buffer suggesting a dual role of HEG as a linker as well as a stabilizer. SPR of bare AuNPs and AuNPsHEG in phosphate buffer is shown in Figure S2. In terms of dispersibility, the concentrated AuNPs-HEG was re-dispersed in water readily. But bare AuNPs did not re-dispersed in water once it aggregated. Morphology of AuNPs-HEG was observed by HR-TEM images. AuNPs-HEG has spherical shape (Figure $3 \mathrm{~b}$ ) and the size of 200 discrete AuNPs-HEG was measured in HR-TEM images with average size of $4.4 \pm 1.0 \mathrm{~nm}$ (Table S1). Bifunctional HEG was used to link AuNPs and Rg3. The -SH functional group was used to attach HEG to AuNPs and the - $\mathrm{COOH}$ functional group was used to bind HEG to the primary hydroxyl groups of Rg3. The HR-TEM image of AuNPs-HEG showed lattice structures originating from a single crystal (Figure $3 \mathrm{e})$. The average distance between two adjacent lattice fringes was measured as $2.355 \AA$.

The plasmonic red-shift was clearly shown in Figure 2 . The distance of two neighboring particles of AuNPs, AuNPs-HEG, and AuNPs-HEG-Rg3 were measured and presented in Table S2. Upon the adsorption of HEG to AuNPs surfaces, the particle distance was changed from $3.1 \pm 1.2 \mathrm{~nm}$ (AuNPs, $515 \mathrm{~nm}$ ) to $2.9 \pm 1.2 \mathrm{~nm}$ (AuNPs-HEG, $521 \mathrm{~nm}$ ) cuasing a 6-nm plasmonic red-shift. However, the conjugation of Rg3 (AuNPs-HEG-Rg3, $523 \mathrm{~nm}$ ) resulted in an increase of the particle distance with a large value of standard deviation $(3.9 \pm 2.8 \mathrm{~nm})$ possibly due to an agglomeration of some particles. The value of particle distance in AuNPs-HEG-Rg3 was varied in the range of $0.7-16.15$ $\mathrm{nm}$ while AuNPs and AuNPs-HEG had a range of 1.0 - $7.2 \mathrm{~nm}$.

For a conjugation of $\mathrm{Rg} 3$ to AuNPs-HEG, solubility of $\mathrm{Rg} 3$ was tested in various organic solvents and DMF was chosen for ester bond formation. Methanol was a good solvent for Rg3; however, alcohol was excluded as it could be a reactant for ester bond formation. When we performed model experiments, $\mathrm{Rg} 3$ formed ester bond with 4-benzyloxy benzoic acid (BBA). Under the conditions of $N, N$-diisopropylcarbodiimide (DIPC)/ 4-( $N, N$ '-dimethylamino)pyridinium-4-toluene sulfonate (DP $\mathrm{TS})$, the reaction yield was better than that of 1-ethyl-3-(3-di-

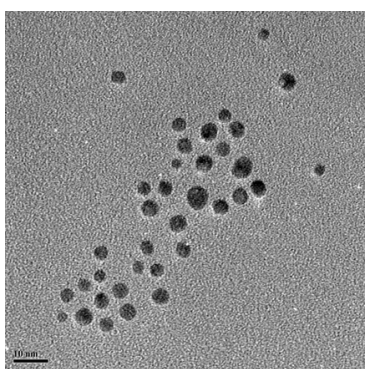

(a)

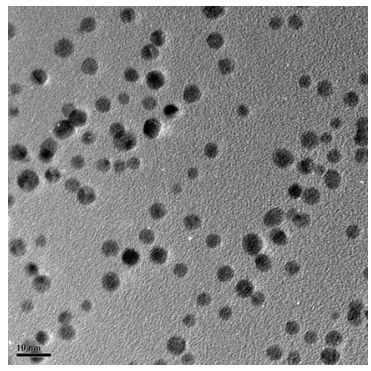

(c)

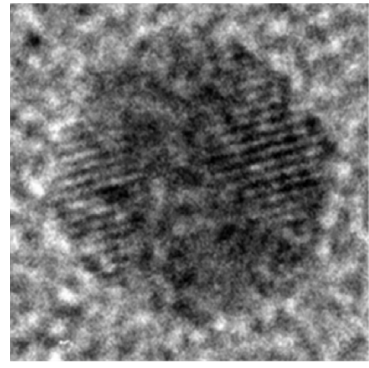

(e)

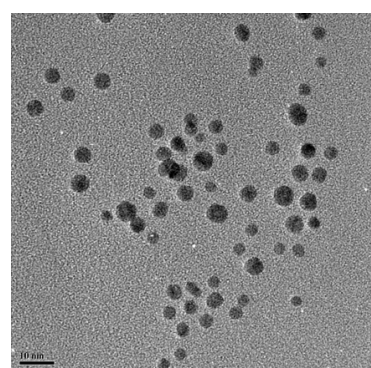

(b)

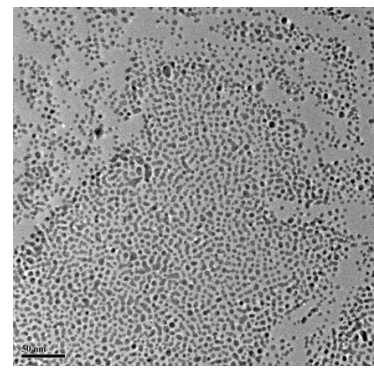

(d)
Figure 3. HR-TEM images of (a) AuNPs (The scale bar corresponds to $10 \mathrm{~nm}$ ), (b) AuNPs-HEG (The scale bar corresponds to $10 \mathrm{~nm}$ ), (c) AuNPs-HEG-Rg3 (The scale bar corresponds to $10 \mathrm{~nm}$ ), (d) AuNPsHEG-Rg3 (The scale bar corresponds to $50 \mathrm{~nm}$ ), and (e) an individual AuNPs-HEG nanoparticle which was enlarged from the image (b). The average distance between two adjacent lattice fringes is $2.355 \AA$.

methylaminopropyl)-carbodiimide (EDC)/4-dimethylaminopyridine (DMAP)/thtraethylamine (TEA). The reaction condition of DIPC/DPTS produced two major products where one or two molecules of BBA bound to one molecule of $\mathrm{Rg} 3$. Molecular ion peaks at $m / z 1018.1\left[\mathrm{Rg} 3+\mathrm{BBA}-\mathrm{H}_{2} \mathrm{O}+\mathrm{Na}\right]^{+}$and $\mathrm{m} / z$ $1228.2\left[\mathrm{Rg} 3+2 \mathrm{BBA}-2 \mathrm{H}_{2} \mathrm{O}+\mathrm{Na}\right]^{+}$were observed in positive ESI mass spectrum (Figure S3). Therefore, DIPC/DPTS was chosen for Rg3 conjugation to AuNPs-HEG. DMF concentration in the reaction mixture was adjusted to $20 \%$ for conducting work-up as the membrane (Nanosep Omega, $30 \mathrm{~K}$ ) is compatible with aqueous solutions containing up to $20 \%$ DMF. Excess reagents and Rg3 were removed by using Nanosep centrifugal device with $70 \%$ methanol washing. Dialysis was selected as another work-up to remove excess $\mathrm{Rg} 3$ and reagents in the reaction mixture. After complete work-up, the final volume of AuNPs-HEG$\mathrm{Rg} 3$ was reduced to $3.6 \mathrm{~mL}$. RP-HPLC-ELSD was conducted to confirm the complete removal of unreacted $\mathrm{Rg} 3$ and excess reagents in final products. No Rg3 was detected in HPLC chromatograms of AuNPs-HEG-Rg3, which confirms that the unreacted $\operatorname{Rg} 3$ had been removed by the work-up process. 
(a)

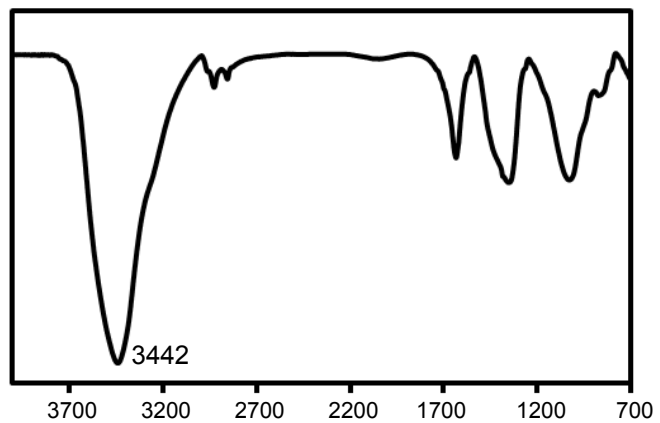

(b)

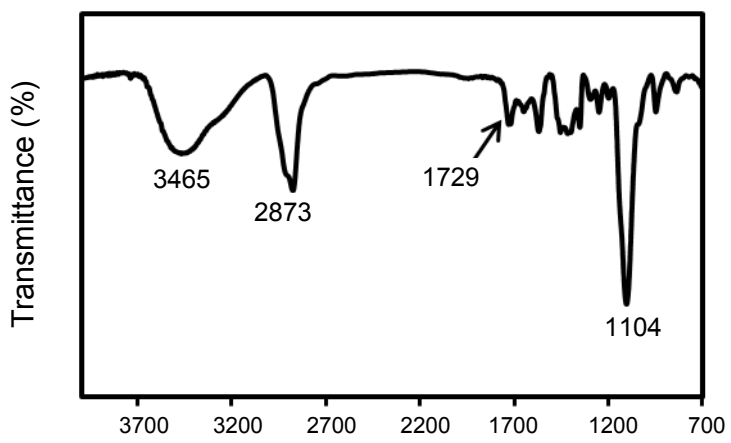

(c)

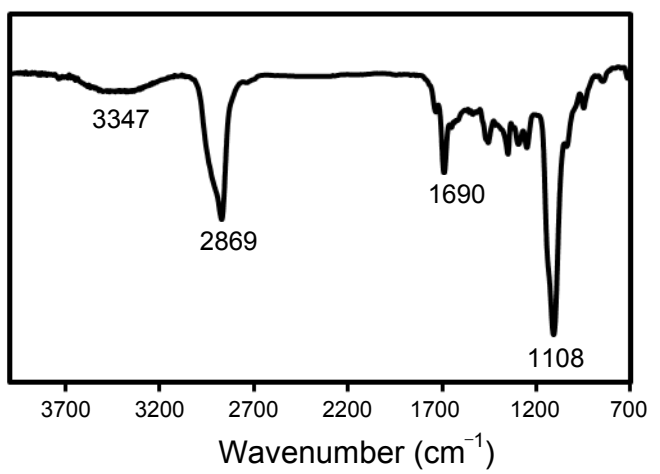

Figure 4. FT-IR spectra of (a) AuNPs, (b) AuNPs-HEG, and (c) AuNPsHEG-Rg3.

Both MALDI-TOF and ESI-MS analyses failed to detect > 100,000 Da of AuNPs-HEG-Rg3, while peaks less than 2,000 were observed (Figure S4). The conjugation between $\operatorname{Rg} 3$ and HEG was also observed in ESI negative ion mode at $\mathrm{m} / z 1686.4$ and $m / z 1592.9$ assigned to [Rg3 $\left.+2 \mathrm{HEG}-2 \mathrm{H}_{2} \mathrm{O}+\mathrm{Na}-2 \mathrm{H}\right]^{-}$and $\left[\mathrm{Rg} 3+2 \mathrm{HEG}-6 \mathrm{H}_{2} \mathrm{O}-\mathrm{H}\right]^{-}$, respectively (Figure $\mathrm{S} 4 \mathrm{a}$ ). In positiveion mode of MALDI-TOF and ESI-MS, sodium adducts of Rg3 were observed at $m / z 807.8$ was $[\mathrm{Rg} 3+\mathrm{Na}]^{+}$, and $\mathrm{m} / z 1591.7$ was $[2 \mathrm{Rg} 3+\mathrm{Na}]^{+}$(Figure S4b). The size of AuNPs-HEG-Rg3 was ranging from $2.1 \mathrm{~nm}$ to $6.7 \mathrm{~nm}$ with average size of $4.7 \pm$ $1.0 \mathrm{~nm}$. The size distribution of each particle (AuNP, AuNPsHEG, and AuNPs-HEG-Rg3) was analyzed using Image J program (NIH free software) on discrete 200 particles of each HRTEM image (Table S1). HR-TEM images showed that modification of gold nanoparticles with $\operatorname{Rg} 3$ did not significantly affect particle size, morphology, and aggregation tendency. The lattice structure was also observed on AuNPs-HEG-Rg3. A Gaussian distribution of AuNPs, AuNPs-HEG, and AuNPs-HEG-Rg3 was observed with narrow size distribution of particles (Figure

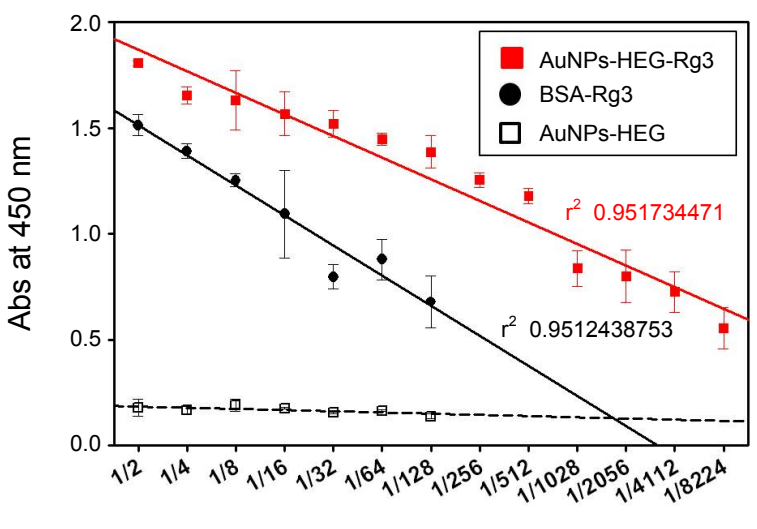

Dilution

Figure 5. ELISA data on AuNPs-HEG-Rg $g_{3}$, AuNPs-HEG (a negative control), and BSA-Rg3 (a positive control). The detailed procedure was described in the methods section.

S5a-c). Information of fingerprint regions of FT-IR provides characteristic peaks from AuNPs, AuNPs-HEG, and AuNPsHEG-Rg3 (Figure 4a-c). The most characteristic band confirming a conjugation between AuNPs-HEG and Rg3 was the band of $\mathrm{C}=\mathrm{O}$. As shown in Figure 4, $\mathrm{C}=\mathrm{O}$ band was observed at 1729 $\mathrm{cm}^{-1}$ (AuNPs-HEG, Figure 4b), and $1690 \mathrm{~cm}^{-1}$ (AuNPs-HEG$\mathrm{Rg} 3$, Figure 4c). Due to the presence of carboxylic acid groups of $\mathrm{HEG}$, a wavenumber of $\mathrm{C}=\mathrm{O}$ was observed at $1729 \mathrm{~cm}^{-1}$ with a broad band of $-\mathrm{OH}$ at $3465 \mathrm{~cm}^{-1}$ (Figure 4b). When Rg3 molecules were conjugated to AuNPs-HEG, the wavenumber of $\mathrm{C}=\mathrm{O}$ was shifted to a low wavenumber of $1690 \mathrm{~cm}^{-1}$ confirming a conjugation. Two bands at $1104 \mathrm{~cm}^{-1}(\mathrm{C}-\mathrm{O})$ and $2873 \mathrm{~cm}^{-1}$ (aliphatic-CH) were appeared in the spectra of AuNPs-HEG. It confirms HEG molecules were attached to AuNPs surfaces. These two bands were shifted at $1108 \mathrm{~cm}^{-1}(\mathrm{C}-\mathrm{O})$ and $2869 \mathrm{~cm}^{-1}$ (aliphatic-CH) when $\mathrm{Rg} 3$ molecules were conjugated to AuNPsHEG. In the case of AuNPs-HEG-Rg3, the average value of zeta potential measurements is $-4.12 \pm 0.38 \mathrm{mV}$ (an average of three measurements: $-3.75,-4.50$, and $-4.10 \mathrm{mV}$ ) while bare AuNPs showed zeta potential of $-18.25 \mathrm{mV}$ (an average of two measurements: -18.02 and $-18.48 \mathrm{mV}$ ). This result demonstrates that HEG linker contributes to the surface charge of the final product by formation of steric layers, which contributes to reduce the aggregation and minimize the opsonization of particles.

The quantity of Rg3 on AuNPs-HEG-Rg3 was determined by ELISA. During ELISA procedures, AuNPs-HEG-Rg3 exhibited excellent stability towards buffers and temperatures $\left(4{ }^{\circ} \mathrm{C}, \mathrm{RT}\right.$, and $\left.37^{\circ} \mathrm{C}\right)$. For ELISA, BSA-Rg3 was prepared according to the previous publication ${ }^{18}$ and the amount of BSA was determined by Bradford assay. One BSA molecule has four Rg3 molecules, ${ }^{18}$ and the numbers of $\operatorname{Rg} 3$ of BSA-Rg3 was calculated from Bradford assay data. Then, ELISA at $450 \mathrm{~nm}$ was performed on AuNPs-HEG-Rg3, BSA-Rg3 (as a positive control), and AuNPs-HEG (as a negative control) with various concentrations to establish calibration curves. The UV at $450 \mathrm{~nm}$ decreased with increasing dilution-fold in both AuNPs-HEGRg3 and BSA-Rg3 (Figure 5). In contrast, AuNPs-HEG showed no change of UV absorbance due to the lack of $\mathrm{Rg} 3$, and the average UV value of AuNPs-HEG was subtracted from that 
of AuNPs-HEG-Rg3 to make a calibration curve of AuNPsHEG-Rg3 (Figure 5). At one fixed UV value of $1(450 \mathrm{~nm})$, we assumed that the number of $\mathrm{Rg} 3$ on both BSA-Rg3 and AuNPs-HEG-Rg3 are the same. Therefore, BSA-Rg3 (2.083 $\mu \mathrm{L}$ ) contained $0.470 \mu \mathrm{g}$ of BSA which was calculated from a calibration curve of Bradford assay. The mole of BSA $(0.470$ $\mu \mathrm{g}$ ) was calculated as $0.71 \times 10^{-11} \mathrm{~mol}$, which equals to $4.27 \times$ $10^{12}$ BSA molecules. From previous reports, one BSA molecule has four Rg3 molecules. ${ }^{18}$ The numbers of Rg3 of BSA-Rg3 was calculated as $1.708 \times 10^{13}$ of $\operatorname{Rg} 3$ molecules $\left(2.63 \times 10^{14}\right.$ of Rg3 molecules/1 mL). Therefore, AuNPs-HEG-Rg3 (0.065 $\mu \mathrm{L}$, UV value 1) has $1.708 \times 10^{13}$ of Rg3 molecules. In this experimental condition, the theoretical number of AuNPs in $1 \mathrm{~mL}$ is $4.2 \times 10^{13}$ particles. Without the loss of AuNPs throughout experimental process, $\mathrm{Rg} 3$ numbers per AuNP is $2.63 \times 10^{14} / 4.2 \times$ $10^{13}=6.26$. In reality, there is a loss of AuNPs during experimental processing and, thus, at least 6 molecules of Rg3 are tethered to the surface of one AuNPs through a HEG linker.

\section{Conclusions}

For many years, ginsenosides have attracted researchers' attention due to their important biological activities. Here, we demonstrate the conjugation of $\mathrm{Rg} 3$, one of ginsenosides from red ginseng, with AuNPs to increase water-solubility of $\mathrm{Rg} 3$. ELISA using Rg3 monoclonal antibody was successfully applied to quantify the number of Rg3 molecules tethered to AuNPs without interference of gold's intrinsic color. Future work remains to be examined including the enhancement of anti-cancer activities of Rg3-conjugated gold nanoparticles. The influence of polyethylene glycol and nanoparticle sizes on biological activities may be important for drug delivery in vivo and further study is necessary.

Acknowledgments. This work was supported by Basic Science Research Program through the National Research Foundation of Korea (NRF) funded by the Ministry of Education, Science and Technology (MEST) (NRF-2008-532-E00032).
This work was partially supported by the research fund for a newly-appointed faculty from Inje University.

\section{References}

1. Daniel, M. C.; Astruc, D. Chem. Rev. 2004, 104, 293.

2. Sperling, R. A.; Gil, P. R.; Zhang, F.; Zanella, M.; Parak, W. J. Chem. Soc. Rev. 2008, 37, 1896.

3. Jain, P. K.; El-Sayed, I. H.; El-Sayed, M. A. Nanotoday 2007, 2, 18.

4. Gibson, J. D.; Khanal, B. P.; Zubarev, E. R. J. Am. Chem. Soc. 2007, 129,11653

5. Bergen, J. M.; von Recum, H. A.; Goodman, T. T.; Massey, A. P.; Pun, S. H.; Macromol. Biosci. 2006, 6, 506.

6. Zhang, G.; Yang, Z.; Lu, W.; Zhang, R.; Huang, Q.; Tian, M.; Li, L.; Liang, D.; Li, C. Biomaterials 2009, 30, 1928.

7. Gu, Y. J.; Che, J.; Lin, C. C.; Lam, Y. W.; Cheng, S. H.; Wong, W. T. Toxicol. Appl. Pharm. 2009, 237, 196.

8. Kuma, S. A.; Peter, Y. A.; Nadeau, J. L. Nanotechnology 2008, $19,1$.

9. Chen, Y. H.; Tsai, C. Y.; Huang, P. Y.; Chang, M. Y.; Cheng, P. C.; Chou, C. H.; Chen, D. H.; Wang, C. R.; Shiau, A. L.; Wu, C. L. Mol. Pharm. 2007, 4, 713.

10. Hosta, L.; Pla-Roca, M.; Arbiol, J.; Lopez-Iglesias, C.; Samitier, J.; Cruz, L. J.; Kogan, M. J.; Albericio, F. Bioconjugate Chem. 2009, 20, 138.

11. Xu, T.; Cui, M.; Xin, Y.; Gu, L.; Jiang, X.; Su, M.; Wang, D.; Wang, W. Chinese Med. J.-Peking 2008, 121, 1394.

12. Luo, X.; Wang, C. Z.; Chen, J.; Song, W. X.; Luo, J.; Tang, N.; He, B. C.; Kang, Q.; Wang, Y.; Du, W.; He, T. C.; Yuan, C. S. Int. J. Oncol. 2008, 32, 975.

13. Zhang, Q.; Kang, X.; Yang, B.; Wang, J.; Yang, F. Cancer Biother. Radio. 2008, 23, 647.

14. Xu, T.; Ying, X.; Cui, M.; Jiang, X.; Gu, L. Chinese Med. J.-Peking 2007, 120, 584.

15. Zhang, Q. H.; Wu, C. F.; Duan, L.; Yang, J. Y. Arch. Toxicol. 2008, 82,117

16. Min, J. K.; Kim, J. H.; Cho, Y. L.; Maeng, Y. S.; Lee, S. J.; Pyun, B. J.; Kim, Y. M.; Park, J. H.; Kwon, Y. G. Biochem. Biophys. Res. Commun. 2006, 349, 987.

17. Song, X.; Zang, L.; Hu, S. Vaccine 2009, 27, 2306.

18. Joo, E. J.; Ha, Y. W.; Shin, H.; Son, S. H.; Kim, Y. S. Biol. Pharm. Bull. 2009, 32, 548 .

19. Ha, Y. W.; Lim, S. S.; Ha, I. J.; Na, Y. C.; Seo, J. J.; Shin, H.; Son, S. H.; Kim, Y. S. J. Chromatogr. A 2007, 1151, 37. 